\title{
Presentations of a numerical semigroup
}

\author{
Belgin Özer ${ }^{1 *}$, Sibel Kanbay ${ }^{1}$ \\ ${ }^{1}$ Gaziantep University,Faculty of Art and Sciences,Department of Mathematics, Gaziantep / Turkey \\ *Corresponding author E-mail: emirhan@gantep.edu.tr
}

\begin{abstract}
In this paper, we mainly study the minimal presentations of numerical semigroups. Moreover, we examine the concept of gluing, complete intersection, catenary degree, elasticity of some numerical semigroups.

Keywords: Catenary Degree; Complete Intersection; Connectedness; Minimal Presentations; Numerical Semigroups.

\section{Introduction}

The object of this paper is to study the presentations of finitely generated numerical semigroups. The study of presentations of numerical semigroups are mainly motivated by its applications to Algebraic Geometry ([1], [2]). Redei in [3] shows that every congruence on $\mathbb{N}^{\mathrm{n}}$ is finitely generated. This result is since then known as Redei's Theorem. Many other authors have given alternative and quite much simpler proofs than his [4 - 7]. Since numerical semigroups are cancellative monoids, a different approach can be chosen to prove Redei's theorem. An important peculiarity of finitely generated cancellative (commutative) monoids is that minimal presentations with respect to set inclusion have minimal cardinality ( [8] ). In this paper, we focus on the computation of a (and in fact all) minimal presentation of a numerical semigroup. The idea comes from Rosales' PhD Thesis ([9]) and was published later in [10]. The cardinality of a numerical semigroup cannot be bounded in terms of its embedding dimension. This follows from Bresinsky's family of embedding dimension four numerical semigroups, which have arbitrarily large minimal presentations ( [11]). In this paper we offer an upper bound for the cardinality of a minimal presentation in terms of the multiplicity of the semigroup.
\end{abstract}

\section{Presentations of a numerical semigroup}

\subsection{Class modulo and quotient set}

Let $X$ be a nonempty set. A binary relation on $X$ is a subset $\sigma \mathrm{X} \times \mathrm{X}$. If $(\mathrm{a}, \mathrm{b}) \in \sigma$, we write $\mathrm{x} \sigma \mathrm{y}$ and we say that $\mathrm{x}$ is $\sigma$ - related with $\mathrm{y}$. If

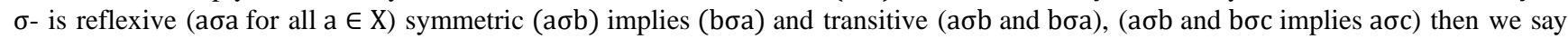
that $\sigma$ is an equivalence binary relation. For every $a \in X$, we define its class modulo $\sigma$ called its $\sigma$ - class as

$[\mathrm{a}]_{\sigma}=\{\mathrm{b} \in \mathrm{X} \mid \mathrm{a} \sigma \mathrm{b}\}$

The set

$\frac{\mathrm{X}}{\sigma}=\left\{[\mathrm{a}]_{\sigma} \mid \mathrm{a} \in \mathrm{X}\right\}$

is the quotient set of $\mathrm{X}$ by $\sigma$, and it is a partition of $\mathrm{X}$.

\subsection{Congruence}

A congruence $\sigma$ is generated by $\rho$ if $\sigma=\operatorname{Cong}(\rho)$. We say that $\rho$ is a system of generators of $\sigma$. A congruence $\sigma$ is finitely generated if there exists a system of generators of $\sigma$ with finitely many elements. A presentation of a finitely generated monoid M is a congruence on Free $(X)$, for some finite set $X$, such that $M \cong \operatorname{Free}(X) / \operatorname{Cong}(\rho)$. We say that $M$ is finitely presented monoid if $\rho$ is finite. Let $X$ be a nonempty set and let $\sigma$ be a congruence on Free(X). An element $(\mathrm{a} \sigma \mathrm{b})$ in $\sigma\{(0,0)\}$ is irreducible if it cannot be expressed as

$(\mathrm{a}, \mathrm{b})=\left(\mathrm{a}_{1}, \mathrm{~b}_{1}\right)+\left(\mathrm{a}_{2}, \mathrm{~b}_{2}\right)$

with, $\left(\mathrm{a}_{1}, \mathrm{~b}_{1}\right),+\left(\mathrm{a}_{2}, \mathrm{~b}_{2}\right) \in \sigma \backslash\{(0,0)\}$. 
Let $\operatorname{Irr}(\sigma)$ be the set of irreducible elements of $\sigma$. Note that $\sigma$ is a submonoid of Free $(\mathrm{X}) \times$ Free(X). Let M be a monoid and $\sigma$ a congruence on $\mathrm{M}$. Then $\frac{\mathrm{M}}{\sigma}$ is a monoid with the operation

$[\mathrm{a}]_{\sigma}+[\mathrm{b}]_{\sigma}=[\mathrm{a}+\mathrm{b}]_{\sigma}$

if $(a, b)$ and $(c, d)$ are in $\sigma$, so is $(a+c, b+d)$, and by reflexivity $(0,0) \in \sigma$. As we see next, $\operatorname{Irr}(\sigma)$ generates $\sigma$ as a monoid.

\subsection{Minimal presentations of a numerical semigroup}

Let $\mathbb{N}$ be the set of non-negative integers and $\mathbb{Z}$ be the set of integers. A numerical semigroup is a finitely generated sub-semigroup $S$ of $\mathbb{N}$ such that $0 \in S$ and $S$ generates $\mathbb{Z}$ as a group. It is known that $S$ is a numerical semigroup if and only if there exist $n_{0}, \ldots, n_{k} \in \mathbb{N} \backslash\{0\}$ such that $\operatorname{gcd}\left\{\mathrm{n}_{0}, \ldots, \mathrm{n}_{\mathrm{k}}\right\}=1$ and $\mathrm{S}=\left\langle\mathrm{n}_{0}, \ldots, \mathrm{n}_{\mathrm{k}}\right\rangle=\left\{\sum_{\mathrm{i}=0}^{\mathrm{k}} \mathrm{m}_{\mathrm{i}} \mathrm{n}_{\mathrm{i}} \mid \mathrm{m}_{\mathrm{i}} \in \mathbb{N}\right\}$.

Every numerical semigroup is a finitely generated cancellative monoid and thus it is finitely presented. We characterize in this section those presentations of numerical semigroups that are minimal. Let $\sigma$ be a congruence on Free $\left(X_{1}, X_{2}, \ldots, X_{n}\right)$ and let $\rho$ be a system of generators of $\sigma$. We say that $\rho$ is a minimal relation if the cardinality of $\rho$ is the least possible among the cardinalities of systems of generators of $\sigma$. Let $S$ be a numerical semigroup minimally generated by $\left\{n_{1}, \ldots, n_{e}\right\}$ and let $X=\left\{x_{1}, \ldots, x_{e}\right\}$ with $x_{i} \neq x_{j}$ for all $i \neq j$.

We say that $\rho$ is a minimal presentation if $\rho$ is a minimal relation of the kernel congruence of

$\varphi:$ Free $\left(\mathrm{x}_{1}, \ldots, \mathrm{x}_{\mathrm{e}}\right) \rightarrow \mathrm{S}, \varphi\left(\mathrm{a}_{1} \mathrm{x}_{1}+\cdots+\mathrm{a}_{\mathrm{e}} \mathrm{x}_{\mathrm{e}}\right)=\mathrm{a}_{1} \mathrm{n}_{1}+\cdots+\mathrm{a}_{\mathrm{e}} \mathrm{n}_{\mathrm{e}}$

In this section,we use $\sigma$ to denote the kernel congruence of $\varphi$. Given $n \in \mathbb{N}$ the set of expressions of $n$ in $S$ is defined as

$\mathrm{Z}_{\mathrm{n}}=\varphi^{-1}(\mathrm{n})=\left\{\mathrm{a}_{1} \mathrm{x}_{1}+\cdots+\mathrm{a}_{\mathrm{e}} \mathrm{x}_{\mathrm{e}} \mid \mathrm{a}_{1} \mathrm{n}_{1}+\cdots+\mathrm{a}_{\mathrm{e}} \mathrm{n}_{\mathrm{e}}=\mathrm{n}\right\}$

For $\mathrm{a}=\mathrm{a}_{1} \mathrm{x}_{1}+\cdots+\mathrm{a}_{\mathrm{e}} \mathrm{x}_{\mathrm{e}} \in$ Free $\left(\mathrm{x}_{1}, \ldots, \mathrm{x}_{\mathrm{n}}\right), \mathrm{b}=\mathrm{b}_{1} \mathrm{x}_{1}+\cdots+\mathrm{b}_{\mathrm{e}} \mathrm{x}_{\mathrm{e}} \in$ Free $\left(\mathrm{x}_{1}, \ldots, \mathrm{x}_{\mathrm{n}}\right)$. Define the dot product of $a$ and $b$ as

$a \cdot b=a_{1} b_{1}+\cdots+a_{e} b_{e}$

We define the following relation on Free $\left(x_{1}, \ldots, x_{n}\right)$. For $a, b \in \operatorname{Free}\left(x_{1}, \ldots, x_{n}\right), a R b$ if either $a=b=0$ or there exist $k_{1}, \ldots, k_{l} \in Z_{n}$ for some $n \in S$ such that $k_{1}=a, k_{l}=b$ and $k_{i} . k_{i+1} \neq 0$ for all $i \in\{1, \ldots, l-1\}$. This is an equivalence binary relation on Free $(X)$. The elements of Free $(X) / R$ are called $R$ - classes. A (nondirected) graph $G$ is a pair $(V, E)$ where $V$ is a set whose elements are known as the vertices of $G$ and $E$ is a subset of $\{\{u, v\} \mid u, v \in V, u \neq v\}$. The unordered pair $\{u, v\}$ will be denoted as $\overline{u v}$, and if it belongs to $E$, then we say that it is an edge of $G$.

A sequence of edges of the form $\overline{v_{o} v_{1}}, \overline{v_{1} v_{2}}, \ldots, \overline{v_{m-1} v_{m}}$ is known as a path of length $m$ connecting them. It is well known that a connected graph with $n$ vertices has at least $n-1$ edges ([12]). A tree is a connected graph with $n$ vertices and $n-1$ edges for some positive integer $n$ (this is one of the many characterizations of a tree).

A subgraph of the graph $G=(V, E)$ is a graph $G^{\prime}=\left(V^{\prime}, E^{\prime}\right)$ such that $V^{\prime} \subseteq V$ and $E^{\prime} \subseteq E$. It is also well known that any connected graph $G$ with $n$ vertices has a subgraph with the same vertices that is a tree. This tree is called the generating tree of $G$.

Let $X$ be a nonempty set, let $P=\left\{X_{1}, \ldots, X_{r}\right\}$ be a partition of $X$ and let $\gamma$ be a binary relation on $X$. The graph associated to $\gamma$ with respect to the partition $P$ is $G_{\gamma}=(V, E)$, where $V=P$ and $\overline{X_{l} X_{j}} \in E$ with $i \neq j$ if there exists $x \in X_{i}$ and $y \in X_{j}$ such that $(x, y) \in \gamma \cup$ $\gamma^{-1}$. Let $n \in \mathbb{N}$ and let $X_{1}, \ldots, X_{r}$ be the $R$ - classes contained in $Z(n)$.

If $\beta$ is a binary relation on Free $\left(x_{1}, \ldots, x_{e}\right)$, we denote by $\beta_{n}=\beta \cap\left(Z_{n} \times Z_{n}\right)$, and by $G_{\beta_{n}}$ the graph associated to the partition $\left\{X_{1}, \ldots, X_{r}\right\}$ of $Z(n)$. As we see next, these graphs are crucial for our characterization of minimal presentations of a numerical semigroup.

Lemma 2.4: Let $n \in \mathbb{N}$. If $\beta$ is a binary relation on Free $\left(x_{1}, \ldots, x_{e}\right)$ generating $\sigma$, then $G_{\beta_{n}}$ is connected.

Theorem 2.5: Let $\beta$ be a binary relation on Free $\left(x_{1}, \ldots, x_{e}\right)$ with $x_{i} \neq x_{j}$ for $i \neq j$. Then $\sigma=$ Cong $(\beta)$ if and only if $G_{\beta_{n}}$ is connected for all $n \in \mathbb{N}$.

Corollary 2.6: Let $S$ be a numerical semigroup. A subset $\beta$ of $\sigma$ is a minimal presentation of $S$ if and only if the cardinality of $\beta_{n}$ equals the number of $R$-classes in $Z(n)$ minus one and $G_{\beta_{n}}$ is connected for all $n \in S$.

Corollary 2.7: The concept of minimal presentation with respect to cardinality and set inclusion coincide for any numerical semigroup. In particular, all minimal presentations have the same cardinality.

\subsection{Computing minimal presentations}

$S=<n_{1}, \ldots, n_{e}>$ and $\sigma$ are as in the preceding section. Let $n$ be an element of $S$. If $X_{1}, \ldots, X_{r}$ are the $R$-classes of $Z(n)$, for all $i \in\{1, \ldots, r\}$ define

$A_{i}=\left\{n_{j} \mid x_{j} \leq x\right.$ for some $\left.x \in X_{i}\right\}$

These sets contain the set of vertices of the different connected components of $G_{n}$. To prove this, we first must show that $\left\{A_{1}, \ldots, A_{r}\right\}$ is a partition of $V_{n}$.

Theorem 2.9: Let $S$ be a numerical semigroup and let $n$ be a nonzero element of $S$. The number of connected components of $G_{n}$ equals the number of $R$-classes in $Z(n)$. 
Proposition 2.10: If $G_{n}$ is not connected, then $n=w+n_{j}$ with $w \in A p\left(S, n_{1}\right) \backslash\{0\}$ and $j \in\{2, \ldots, e\}$.

Example 2.11: Assume now that $S$ is minimally generated by $\left\{n_{1}, n_{2}, n_{3}\right\}$. For every $r \in\{1,2,3\}$, define

$c_{r}=\min \left\{c \in \mathbb{N}\{0\} \mid k_{n_{r}} \in<n_{s}, n_{t}>,\{r, s, t\}=\{1,2,3\}\right\}$

For $n \in\left\{c_{1} n_{1}, c_{2} n_{2}, c_{3} n_{3}\right\}$, the graph $G_{n}$ is not connected,and these are the only elements in $S$ fulfilling this condition. We distinguish three cases.

If $c_{1} n_{1}=c_{2} n_{2}=c_{3} n_{3}$, then $\left\{\left(c_{1} x_{1}, c_{2} x_{2}\right),\left(c_{1} x_{1}, c_{3} x_{3}\right)\right\}$ is a minimal presentation for $S$.

Assume now that $c_{1} n_{1} \neq c_{2} n_{2}=c_{3} n_{3}$ (we omit the other similar cases). If $c_{1} n_{1}=\lambda n_{2}+\mu n_{3}$ with $\lambda, \mu \in \mathbb{N}$, then

$\left\{\left(c_{1} x_{1}, \wedge x_{2}+\mu x_{3}\right),\left(c_{2} x_{2}, c_{3} x_{3}\right)\right\}$ is a minimal presentation for $S$. If the cardinality of $\left\{c_{1} n_{1}, c_{2} n_{2}, c_{3} n_{3}\right\}$ is there, then suppose that

$c_{1} n_{1}=r_{12} n_{2}+r_{13} n_{3}, c_{2} n_{2}=r_{21} n_{1}+r_{23} n_{3}, c_{3} n_{3}=r_{31} n_{1}+r_{32} n_{2}$

for some nonnegative integers $r_{i j}$. Then

$\left\{\left(c_{1} x_{1}, r_{12} x_{2}+r_{13} x_{3}\right),\left(c_{2} x_{2}, r_{21} x_{1}+r_{23} x_{3}\right),\left(c_{3} x_{3}, r_{31} x_{1}+r_{32} x_{2}\right)\right\}$

is a minimal presentation for $S$.

Theorem 2.12: Let $S$ be a numerical semigroup minimally generated by $\left\{n_{1}, \ldots, n_{e}\right\}$. The cardinality of any minimal presentation for $S$ is less than or equal to

$\frac{\left(2 n_{1}-e+1\right)(e-2)}{2}+1$

Corollary 2.13: Let $S$ be a numerical semigroup. The cardinality of any minimal presentation for $S$ is less than or equal to

$\underline{m(S) \cdot(m(S)-1)}$

\section{Factorizations and divisibility}

For a factorization $x=\left\{x_{1}, \ldots, x_{n}\right\}$ of is its length is defined as $|x|=x_{1}+\cdots+x_{p}$.

The set of $s$ is $L(s)=\{|x| \mid x \in Z(s)\}$.

Theorem 3.1: Let $S$ be a numerical semigroup minimally generated by $\left\{n_{1}, \ldots, n_{p}\right\}$ with $n_{1}<\cdots<n_{p}$. Then

$\rho(S)=\frac{n_{p}}{n_{1}}$

Assume that $L(s)=\left\{l_{1}<\cdots<l_{k}\right\}$. Define the delta set of $s$ as

$\Delta(s)=\left\{l_{2}-l_{1}, \ldots, l_{k}-l_{k-1}\right\}$

and if $k=1, \Delta(s)=\emptyset$

The delta set of $S$ is defined as

$\Delta(S)=\bigcup_{s \in S} \Delta(s)$.

Theorem 3.2: Let $S$ be a numerical semigroup and let $\sigma$ be a presentation of $S$. Then

$\min \Delta(S)=\operatorname{gcd}\{|a-b| \mid(a, b) \in \sigma\}$

Definition 3.3: Let $S$ be a numerical semigroup and $m \in S, x, y \in Z_{S}(m), M \in \mathbb{N}$. In this case, an $M$-chain from $x$ to $y$ is such that $x_{1}=x, x_{i}=y$ and $d\left(x_{j}, x_{j+1}\right) \leq M$ for each $j \in\{1, \ldots, i-1\}$. The multiplier of $m$ is $x_{1}, \ldots, x_{i} \in Z_{S}(m)[13]$.

Definition 3.4: Let $S$ be a numerical semigroup and $m \in S, x, y \in Z_{S}(m), M \in \mathbb{N}$. In this case, the catenary degree of $m$ is the smallest of the $M$-chains that exist from $x$ to $y$. In addition, the catenary grade of $m$ is indicated by $c(m)$ and the catenary degree set of the numerical half group of $S$ is indicated by $C(S)=\{c(s) \mid s \in S\}$ [13]. 


\section{Minimal presentations}

\subsection{Generators and relations}

Let $S$ be a numerical semigroup minimally generated by $\left\{n_{1}, n_{2}, \ldots, n_{p}\right\}$. Then the monoid morphism

$\varphi: \mathbb{N}^{p} \rightarrow S, \varphi\left(a_{1}, \ldots, a_{p}\right)=\sum_{i=1}^{p} a_{i} n_{i}$

known as the factorization homomorphism of $S$, is an epimorphism, and consequently $S$ is isomorphic to $\mathbb{N}^{p} /$ ker $\varphi$, where $k e r \varphi$ is the kernel congruence of $\varphi$ :

$\operatorname{ker} \varphi=\left\{(a, b) \in \mathbb{N}^{p} \times \mathbb{N}^{p} \mid \varphi(a)=\varphi(b)\right\}$

Notice that for groups, vector spaces, rings...the kernel is defined by the elements mapping to the identity element. This is because there we have inverses and from $f(a)=f(b)$ we get $f(a-b)=0$.

Given $\tau \subset \mathbb{N}^{p} \times \mathbb{N}^{p}$, the congruence generated by $\tau$ is the smallest congruence on $\mathbb{N}^{p}$ containing $\tau$, that is, it is the intersection of all congruences containing $\tau$. We denote by $\operatorname{cong}(\tau)$ the congruence generated by $\tau$. Accordingly, we say that $\tau$ is a generating system of a congruence $\sigma$ on $\mathbb{N}^{p}$ if $\operatorname{cong}(\tau)=\sigma$. The congruence generated by a set is precisely the reflexive, symmetric, transitive closure (this would just maket he closure an equivalence relation), to which we adjoin all pairs $(a+c, b+c)$ whenever $(a, b)$ is in the closure; so that the resulting relation becomes a congruence. This can be formally written as follows.

Proposition 4.2: Let $\rho \subseteq \mathbb{N}^{p} \times \mathbb{N}^{p}$. Define $\rho^{0}=\rho \cup\{(b, a) \mid(a, b) \in \rho\} \cup\left\{(a, a) \mid a \in \mathbb{N}^{p}\right\}, \rho^{1}=(v+u, w+u),(v, w) \in \rho^{0}$,

$u \in \mathbb{N}^{p}$. Then $\operatorname{cong}(\rho)$ is the set of pairs $(v, w) \in \mathbb{N}^{p} \times \mathbb{N}^{p}$ such that there exist $k \in \mathbb{N}$ and $v_{0}, \ldots, v_{k} \in \mathbb{N}^{p}$ with $v_{0}=v, v_{k}=w$ and $\left(v_{i}, v_{i+1}\right) \in \rho^{1}$ for all $i \in\{0, \ldots, k-1\}$.

\subsection{Free numerical semigroups}

In this section, we recall the concept of free numerical semigroup and show how to compute a presentation for these semigroups. Let $S$ be the semigroup generated by $\left\{r_{0}, \ldots, r_{h}\right\}$. Set $d_{1}=r_{0}$ and $d_{k}=\operatorname{gcd}\left(d_{k-1}, r_{k-1}\right)$ for all $k \in\{2, \ldots, h+1\}$ (whence $\left.d_{h+1}=1\right)$, and define $e_{k}=d_{k} / d_{k+1}$. Recall that $S$ is free for the arrangement $\left(r_{0}, \ldots, r_{h}\right)$ of generators if for all $k \in\{1, \ldots, h\}$ :

i) $\quad e_{k}>1$,

ii) $\quad e_{k} r_{k}$ belongs to the semigroup generated by $\left\{r_{o}, \ldots, r_{k-1}\right\}$.

Definition 4.4: Let $S$ be a numerical semigroup and let $n$ be one of its nonzero elements. The apery set of $n$ in $S$ is

$A p(S, n)=\{s \in S \mid s-n \notin S\}$

Definition 4.5: Let $S$ be a numerical semigroup. The cardinality of a minimal set of generators of $S$ is called the embedding dimension of S. We denote it by $e(S)$.

Definition 4.6: Let $^{*}=S \backslash\{0\}$. The smallest nonzero element of $S$ is called the multiplicity of $S, m(S)=m i n S^{*}$.

Definition 4.7: The idea of gluing is the following, A set of positive integers $A$, which is usually taken as the set of generators of a monoid, is the gluing of $A_{1}$ and $A_{2}$ if $\left\{A_{1}, A_{2}\right\}$ is a partition of $A$ and the monoid generated by $A$ admits a presentation in which some relators only involve generators in $A_{1}$, other relators only involve generators in $A_{2}$ and there is only one element in this presentation relating elements in $A_{1}$ with elements in $A_{2}$. In order to formalize this definition we need to recall and introduce some notation.

Let $A=\left\{m_{1}, \ldots, m_{r}\right\}$ be a subset of positive integers, $X=\left\{x_{1}, \ldots, x_{r}\right\} . \varphi:$ Free $(X) \rightarrow \mathbb{N}$ the monoid homomorphism.

$\varphi\left(a_{1} x_{1}+\cdots+a_{r} x_{r}\right)=a_{1} m_{1}+\cdots+a_{r} m_{r}$. Denote by $\sigma$ the kernel congruence of $\varphi$, that is, a $\sigma b$ if and only if $\varphi(a)=\varphi(b)$. For $B \subseteq$ $A$, set $X_{B}=\left\{x_{i} \mid m_{i} \in B\right\}$. Then Free $\left(X_{B}\right) \subseteq$ Free $(X)$. We define $\varphi_{B}$ and $\sigma_{B}$ accordingly. Note that $\sigma_{B} \subseteq \sigma$. With this notation is now easy to express the concept of gluing. Let $\left\{A_{1}, A_{2}\right\}$ be a partition of $A$. We say that $A$ is the gluing of $A_{1}$ and $A_{2}$ if there exists a system of generators $\rho$ of $\sigma$ such that $\rho=\rho_{1} \cup \rho_{2} \cup\{(a, b)\}$ with $\rho_{1} \subseteq \sigma_{A_{1}}, \rho_{2} \subseteq \sigma_{A_{2}}, 0 \neq a \in$ Free $\left(X_{A_{1}}\right)$ and $0 \neq b \in$ Free $\left(X_{A_{2}}\right)$.

Definition 4.8: Let $S$ be a numerical semigroup and let $n$ be one of its nonzero elements. If one of the minimal representatives of $S$ if the number of elements $e(S)-1, S$ is called the complete intersection.

\section{Examples}

\subsection{Example}

Let $\boldsymbol{S}=\langle\mathbf{6}, \mathbf{4}, \mathbf{1 7}\rangle$

$A p(S, 4)=\{0,6,17,23\}$

Betti set $\{12,23,29,34,40\}$. 
Hence, we look at the graphs $G_{n}$, with $n$ in the set $\{12,23,29,34,40\}$.

\begin{tabular}{lll}
\hline Graph & Connected components & Relations \\
\hline$G_{12}$ & $\{6\},\{4\}$ & $\left\{2 x_{1}, 3 x_{2}\right\}$ \\
$G_{23}$ & $\{6,17\}$ & $\left\{x_{1}+x_{3}\right\}$ \\
$G_{29}$ & $\{6,17\},\{4,17\}$ & $\left\{2 x_{1}+x_{3}, 3 x_{2}+x_{3}\right\}$ \\
$G_{34}$ & $\{6,4\},\{17\},\{6,4\}$ & $\left\{5 x_{1}+x_{2}, 2 x_{3}, x_{1}+7 x_{2}\right\}$ \\
$G_{40}$ & $\{4\},\{6,17\},\{6,4\}$ & $\left\{10 x_{2}, x_{1}+2 x_{3}, 4 x_{1}+4 x_{2}\right\}$ \\
\hline
\end{tabular}

Hence a minimal presentation for $S$ is

$\rho=\{((2,0,0),(0,3,0)),((5,1,0),(0,0,2))\}$

$\mathrm{v}(S)=2, e(S)=3, \mathrm{v}(S)=e(S)-1$ it is a complete intersection, since $A_{1}=\langle 6,4\rangle$ ve $A_{2}=\langle 17\rangle$ are gluings of $S . L(12)=\{2,3\}$,

$\Delta(12)=\{1\}, \rho(12)=\frac{3}{2}, L(23)=\{2\}, \Delta(23)=\emptyset, L(29)=\{3,4\}$

$\Delta(29)=\{1\}, \rho(29)=\frac{4}{3}, L(34)=\{2,6,7,8\}, \Delta(34)=\{1,4\}, \rho(34)=4, L(40)=\{3,7,8,9,10\}$

$\Delta(40)=\{1,4\}, \rho(40)=\frac{10}{3}, \Delta(S)=\bigcup_{s \in S} \Delta(s)=\{1,4\}, \rho(S)=\sup \{\rho(s) \mid s \in S\}=4$

$G_{34}$

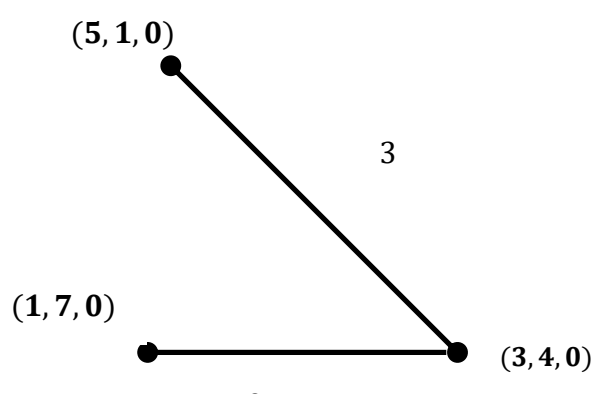

3

Catenary degree of 34 is 3 .

\section{Example 5.2.}

Let $S=\langle 5,7,11,13\rangle$

$A p(S, 5)=\{0,7,11,13,14\}$,

Betti set $\{14,18,20,21,22,24,25,26,27\}$.

Hence, we look at the graphs $G_{n}$, with $n$ in the set $\{14,18,20,21,22,24,25,26,27\}$.

\begin{tabular}{lll}
\hline Graph & Connected components & Relations \\
\hline$G_{14}$ & $\{7\}$ & $\left\{2 x_{2}\right\}$ \\
$G_{18}$ & $\{5,13\},\{7,11\}$ & $\left\{x_{1}+x_{4}, x_{2}+x_{3}\right\}$ \\
$G_{20}$ & $\{5\},\{7,13\}$ & $\left\{4 x_{1}, x_{2}+x_{4}\right\}$ \\
$G_{21}$ & $\{7\},\{5,11\}$ & $\left\{3 x_{2}, 2 x_{1}+x_{3}\right\}$ \\
$G_{22}$ & $\{5,7\},\{11\}$ & $\left\{3 x_{1}+x_{2}, 2 x_{3}\right\}$ \\
$G_{24}$ & $\{11,13\},\{5,7\}$ & $\left\{x_{3}+x_{4}, 2 x_{1}+2 x_{2}\right\}$ \\
$G_{25}$ & $\{5\},\{5,7,13\},\{7,11\}$ & $\left\{5 x_{1}, x_{1}+x_{2}+x_{4}, 2 x_{2}+x_{3}\right\}$ \\
$G_{26}$ & $\{13\},\{5,11\},\{7,11\}$ & $\left\{2 x_{4}, 3 x_{1}+x_{3}\right\}$ \\
$G_{27}$ & $\{5,7\},\{7,13\},\{5,11\}$ & $\left\{4 x_{1}+x_{2}, 2 x_{2}+x_{4}, x_{1}+2 x_{3}\right\}$ \\
\hline
\end{tabular}

Hence a minimal presentation for $S$ is

$\rho=\{((1,0,0,1),(0,1,1,0)),((4,0,0,0),(0,1,0,1)),((0,3,0,0),(2,0,1,0)),((3,1,0,0),(0,0,2,0))$

$((0,0,1,1),(2,2,0,0)),((5,0,0,0),(0,2,1,0)),((0,0,0,2),(3,0,1,0)),((0,2,0,1),(1,0,2,0))\}$

$\mathrm{v}(S)=8, e(S)=4$, since $\mathrm{v}(S) \neq e(S)-1$ it is not complete intersection. $L(14)=\{2\}, \Delta(14)=\emptyset$

$L(18)=\{2\}, \Delta(18)=\emptyset, L(20)=\{2,4\}, \Delta(20)=\{2\}, \rho(20)=2, L(21)=\{3\}$,

$\Delta(21)=\emptyset, L(22)=\{2,4\}, \Delta(22)=\{2\}, \rho(22)=2, L(24)=\{2,4\}, \Delta(22)=\{2\}, \rho(22)=2$,

$L(26)=\{2,4\}, \Delta(26)=\{2\}, \rho(26)=2, L(27)=\{3,5\}, \Delta(27)=\{2\}, \rho(27)=\frac{5}{3}$,

$\Delta(S)=\bigcup_{s \in S} \Delta(s)=\{2\}, \rho(S)=\sup \{\rho(s) \mid s \in S\}=2$. 
$G_{25}$

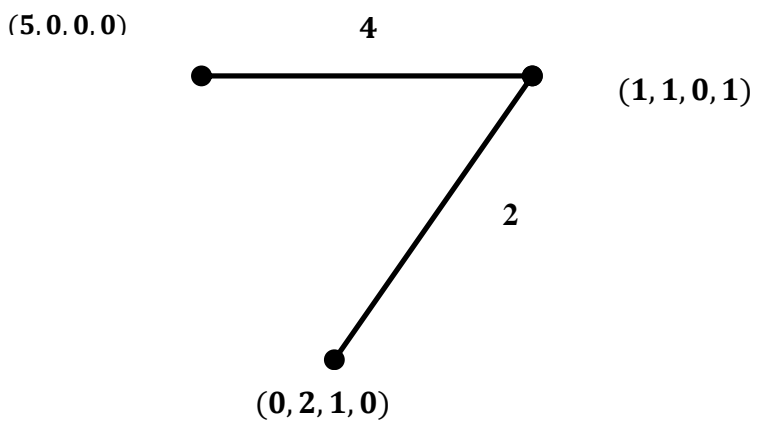

Catenary degree of 25 is 4 .

\section{Example 5.3.}

Let $S=\langle 2,3\rangle$

$A p(S, 2)=\{0,3\}$

Betti Elements

$\{6\}$

Hence, we look at the graphs $G_{n}$, with $n$ in the set $\{6\}$.

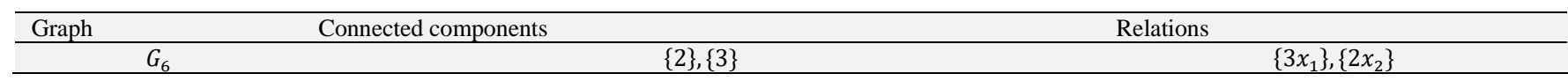

Hence a minimal presentation for $S$ is

$\rho=\{((3,0),(0,2))\}$

$\mathrm{v}(S)=1, e(S)=2$, since $\mathrm{v}(S)=e(S)-1$ it is not complete intersection. $A_{1}=\langle 2\rangle$ and $A_{2}=\langle 3\rangle$ is a gluing of $S . L(6)=\{2,3\}, \Delta(6)=$ $\{1\}, \rho(6)=\frac{3}{2}, \Delta(S)=\bigcup_{s \in S} \Delta(s)=\{1\}, \rho(S)=\sup \{\rho(s) \mid s \in S\}=\frac{3}{2}$

$G_{6}$

\section{Example 5.4.}

Let $S=\langle 3,5,7\rangle$.

$\operatorname{Ap}(S, 3)=\{0,5,7\}$

Betti Elements

$\{10,12,14\}$

Hence, we look at the graphs $G_{n}$, with $n$ in the set $\{10,12,14\}$.

\begin{tabular}{cccc}
\hline Graph & \multicolumn{2}{c}{ Connected components } & Relations \\
\hline & $G_{10}$ & $\{3,7\},\{5\}$ & $\left\{x_{1}+x_{3}\right\},\left\{2 x_{2}\right\}$ \\
& $G_{12}$ & $\{3\},\{5,7\}$ & $\left\{4 x_{1}\right\},\left\{x_{2}+x_{3}\right\}$ \\
& & $\{3,5\},\{7\}$ & $\left\{3 x_{1}+x_{2}\right\},\left\{2 x_{3}\right\}$ \\
\hline
\end{tabular}

Hence a minimal presentation for $S$ is

$\rho=\{((1,0,1),(0,2,0)),((4,0,0),(0,1,1)),((3,1,0),(0,0,2))\}$

$\mathrm{v}(S)=3, e(S)=3$, since $\mathrm{v}(S) \neq e(S)-1$ it is not complete intersection. $L(10)=\{2\} \Delta(10)=\emptyset$,

$L(12)=\{2,4\}, \Delta(12)=\{2\}, \rho(12)=2, L(14)=\{2,4\}, \Delta(14)=\{2\}, \rho(14)=2$,

$\Delta(S)=\bigcup_{s \in S} \Delta(s)=\{2\}, \rho(S)=\sup \{\rho(s) \mid s \in S\}=2$ 
$G_{12}$

\section{Affine semigroups having a unique betti element}

\section{Example 6.1.}

Let $S=\left\langle\mathbb{N}^{2}\right\rangle$

$A=\left(\begin{array}{lll}2 & 0 & 1 \\ 0 & 2 & 1\end{array}\right), S_{1}=N\left(\begin{array}{l}2 \\ 0\end{array}\right)+N\left(\begin{array}{l}0 \\ 2\end{array}\right), S_{2}=N\left(\begin{array}{l}1 \\ 1\end{array}\right), \operatorname{Betti}(S)=\operatorname{Betti}\left(S_{1}\right) \cup \operatorname{Betti}\left(S_{2}\right) \cup\{d\}$.

If $S$ is a free affine semigroup,then $\operatorname{Betti}(S)=\emptyset, d \in S_{1} \cap\left\{S_{2} \backslash\{0\}\right\}, d=\left(\begin{array}{l}2 \\ 2\end{array}\right)$,

$\operatorname{Betti}\left(S_{1}\right)=\operatorname{Betti}\left(S_{2}\right)=\emptyset$, since $S$ is a gluing of $S_{1}$ and $S_{2}, \operatorname{Betti}(S)=d=\left(\begin{array}{l}2 \\ 2\end{array}\right)$

Hence a minimal presentation for $S$ is

$\rho=\{((1,1,0),(0,0,2))\}$

$A$ is a matrix of type $r \times p, \mathrm{v}(S)=1$, since $\mathrm{v}(S)=\rho-\operatorname{rank}(A)=3-2=1$ it is a complete intersection, $L\left(\begin{array}{l}2 \\ 2\end{array}\right)=\left\{\begin{array}{l}2 \\ 2\end{array}, \Delta\left(\begin{array}{l}2 \\ 2\end{array}\right)=\emptyset\right.$, $\Delta(S)=\bigcup_{s \in S} \Delta(s)=\emptyset$.

Example 6.2.

$A=\left(\begin{array}{llllll}1 & 1 & 1 & 0 & 0 & 0 \\ 1 & 0 & 0 & 1 & 1 & 0 \\ 0 & 1 & 0 & 1 & 0 & 1 \\ 0 & 0 & 1 & 0 & 1 & 1\end{array}\right)$

$S_{1}=\mathrm{N}\left(\begin{array}{l}1 \\ 1 \\ 0 \\ 0\end{array}\right)+\mathrm{N}\left(\begin{array}{l}0 \\ 0 \\ 1 \\ 1\end{array}\right)$

$S_{2}=\mathrm{N}\left(\begin{array}{l}1 \\ 0 \\ 1 \\ 0\end{array}\right)+\mathrm{N}\left(\begin{array}{l}0 \\ 1 \\ 0 \\ 1\end{array}\right)$

$S_{3}=\mathrm{N}\left(\begin{array}{l}1 \\ 0 \\ 0 \\ 1\end{array}\right)+\mathrm{N}\left(\begin{array}{l}0 \\ 1 \\ 1 \\ 0\end{array}\right)$

$S$ is a gluing of $S_{1}, S_{2}$ and $S_{3}$. Betti $(S)=d=\{(1,1,1,1)\}$.

Hence a minimal presentation for $S$ is

$\rho=\{((1,0,0,0,0,1),(0,1,0,0,1,0),(0,0,1,1,0,0))\}$

$A, r \times p$ türünde bir matris. $\mathrm{v}(S)=1$, since $\mathrm{v}(S) \neq \rho-\operatorname{rank}(A)$ it is not a complete intersection.

$L((1,1,1,1))=\{2\}, \Delta((1,1,1,1))=\emptyset, \Delta(S)=\bigcup_{s \in S} \Delta(s)=\emptyset$. 
Table 1: Data Table of Numerical Semi-Groups in Examples

\begin{tabular}{|c|c|c|c|c|c|c|}
\hline Semigroup & Betti elements & Gluing & $\begin{array}{l}\text { Complete } \\
\text { Intersection }\end{array}$ & Elasticity & $\begin{array}{l}\text { Catenary } \\
\text { Degree }\end{array}$ & Delta \\
\hline$S=\langle 6,4,17\rangle$ & $\{12,23,29,34,4\}$ & $\begin{array}{l}A_{1}=<6,4> \\
A_{2}=<17>\end{array}$ & yes & 4 & 6 & $\{1,4\}$ \\
\hline $\mathrm{S}=\langle 5,7,11,13\rangle$ & $\{14,18,20,21,22,24,25,26,27\}$ & Don't writing & no & 2 & 5 & $\{2\}$ \\
\hline $\mathrm{S}=\langle 2,3\rangle$ & $\{6\}$ & $\begin{array}{l}A_{1}=<2> \\
A_{2}=<3>\end{array}$ & yes & $3 / 2$ & 3 & $\{1\}$ \\
\hline $\mathrm{S}=\langle 3,5,7\rangle$ & $\{10,12,14\}$ & Don't writing & no & 2 & 4 & $\{2\}$ \\
\hline
\end{tabular}

\section{Acknowledgement}

Our thanks to the Prof.Pedro Garcia Sanchez who have contributed towards development of the article.

\section{References}

[1] V. Barucci, Valentina Numerical semigroup algebras, in Multiplicative ideal theory in commutative algebra, 39-53, Springer, New York, 2006. "available online : https://doi.org/10.1007/978-0-387-36717-0 3".

[2] V. Barucci, D. E. Dobbs, M.Fontana, Maximality Properties in Numerical Semigroups and Applications to One-Dimensional Analytically Irreducible Local Domains, Memoirs of the Amer. Math. Soc. 598 (1997). "available online : https://doi.org/10.1090/memo/0598"

[3] L. Redei, The theory of finitely generated commutative semigroups, Pergamon, Oxford-Edinburgh-New York, 1965.

[4] P. Freyd, Redei's finiteness theorem for commutative semigroups, Proc. Amer. Math. Soc. 19 (1968), 1003. "available online : https://doi.org/10.1090/S0002-9939-1968-0227290-4".

[5] P. A. Grillet, A short proof of Redei’s theorem, Semigroup Forum 46 (1993), 126-127."available online : https://doi.org/10.1007/BF02573555".

[6] J. Herzog, Generators and relations of abelian semigroups and semigroup rings, Manuscripta Math. 3 (1970), 175-193. "available online : https://doi.org/10.1007/BF01273309",

[7] J. C. Rosales, Function minimum associated to a congruence on integral n-tuple space, Semigroup Forum 51 (1995) 87-95. "available online : https://doi.org/10.1007/BF02573622".

[8] J. C. Rosales, P.A. Garcia-Sanches, J.M. Urbano-Blanco, On presentations of commutative monoids, Internat. J. Algebra Comput. 9 (1999), no. 5, 539-553. "available online : https://doi.org/10.1142/S0218196799000333".

[9] J. C. Rosales, Semigrupos numericos, Tesis Doctoral, Universidad de Granada, Spain, 2001.

[10] J. C. Rosales, An algorithmic method to compute a minimal relation for any numerical semigroup, Internat. J. Algebra Comput. 6 (1996), no. 4, 441-455." available online : https://doi.org/10.1142/S021819679600026X".

[11] H. Bresinsky, On prime ideals with generic zeo $x_{i}=t^{n_{i}}$, Proc. Amer. Math. Soc. 47 (1975), 329-332. “available online : https://doi.org/10.2307/2039739".

[12] D. Narsingh, Graph Theory with Applications to Engineering and Computer Science, Prentice Hall Series in Automatic Computation, 1974

[13] (Assi ve Garcia-Sanchez, 2014; Chapman ve ark., 2016; O’Neil ve ark., 2016) 